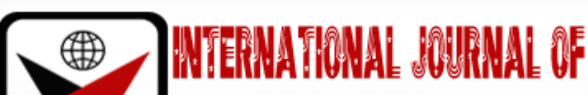

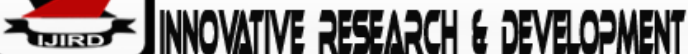

ISSN 2278-0211 (Online)

\section{A Low-Cost Telephony System for Small/Medium Scale Businesses in Nigeria: An Implementation of VoIP on a LAN Using Mini SIP Server}

\begin{tabular}{|c|}
\hline Nwaogwugwgu, Ibeabuchi Benjamin \\
Lecturer, Department of Computer Science \\
Godfrey Okoye University, Enugu, Nigeria \\
Amachundi, Samuel Adda \\
Lecturer, Department of Computer Science, \\
Federal University, Wukari, Taraba State, Nigeria \\
Nandom, Sumayyah Sophie \\
Lecturer, Department of Computer Science, \\
Federal University, Wukari, Taraba State, Nigeria \\
Nze Chiedozie Victor \\
Graduate Assistant, Department of Computer Science, \\
Godfrey Okoye University, Enugu, Nigeria \\
\hline
\end{tabular}

\begin{abstract}
VoIP (Voice over Internet Protocol) is a technology that transforms your voice into a digital signal, allowing you to make a call from a computer, a VoIP phone, or other data-driven devices. Simply stated, it is an internet-based telecommunications service. Traditional telephony services can now be distributed over computer networks using packet-switched protocols thanks to VoIP technology. Any VoIP-compatible network, such as a local area network, can carry VoIP packets (LAN). Switching to VoIP could save you money on communication services. With a VoIP service, long-distance and international calls are normally secure. This study simulated VoIP technology over a typical school local area network, allowing voice and video calls to be made across the network. This framework was evaluated and developed using the Object-Oriented Methodology. The system was made up of two main components: software and hardware. A miniSIP server and a client softphone named Zoiper make up the software portion. The hardware part is made up of two laptops, a phone, an Ethernet cable and a wireless router. The miniSIP server helps the network administrator create local user and assign them call number. The Zoiper softphone (which is a client software), is used to connect the miniSIP server for voice or video communication. When a client places a call over the LAN network using the Zoiper softphone, the Zoiper client software connects the miniSIP server on the network to indicate that a call has been placed, then the miniSIP server connects both clients (the caller and the receiver) using each client or user's IP address to establish communication between both clients. During voice communication between both clients, each has option to request for a video call in the middle of the voice call which can be accepted or declined by the client receiving the request. A real-time testing of this system was carried out and it proved to be successful.
\end{abstract}

Keywords: VoIP, network, communication, LAN, telecommunication

\section{Introduction}

The wired telecommunications system over which landline telephone calls are made is known as the Public Switched Telephone Network (PSTN), also known as Plain Old Telephone Service (POTS). It's also a connection-oriented, circuit-switched network that creates a dedicated channel (or circuit) for the duration of a transmission (Dave Roos, 2008). To connect one phone to another, the phone call is routed through numerous switches operating on a local, regional, national or international level (Dave Roos, 2008).

In the early days, phone calls traveled as analog signals across copper wire. Every phone call needed its own dedicated copper wire connecting the two phones. The operators sat at a switchboard, literally connecting one piece of copper wire to another so that the call could travel across town or across the country. When two telephones are connected, analog voice data is transmitted over the copper wires of the PSTN. The voice data is then converted into electrical signals which are eventually routed in the switching centers. Finally, a connection is made and communication is possible (Dave Roos, 2008).

Long-distance calls were relatively costly because you were renting the use of a very long piece of copper wire each time you called. Since the late 1800s, this is the system that has been commonly used (Zhang, 2006). For centuries, 
these underground copper wires provided companies and households with a secure means of connecting with everyone everywhere in the world. Although the PSTN was effective and does a good job, many business drivers strived to change it to a new network-voice over a data network.

This means that voice calls, like normal phone calls, are sent over packet-switched data networks, which transform the voice signal from the phone into a digital signal that travels over the internet and then translates it back at the other end, enabling you to talk to someone with a regular phone number. Data is now running on top of networks that were developed to efficiently carry voice (Zhang, 2006). However, due to variable use of bandwidth and a need for higher bandwidth, scalability of traditional circuit-switched networks is limited and call charges are higher. These gave rise to VoIP. Voice over Internet Protocol (VoIP) transmission began in 1973 as a result of the experimental Network Voice Protocol invented for the Advanced Research Projects Agency Network (ARPANET) (Fasiku, 2018). However, it wasn't until 1995 that the first Internet Phone Software (Vocaltec) appeared. Vocaltec brought to market the first internet phone software called the Internet Phone (Fasiku, 2018). The Vocaltec software compressed the voice signal, translated it into digital packets, and distributed it over the Internet. One of the main features of the VoIP technology is that it may be deployed using a centralized or a distributed architecture (Fasiku, 2018). With this new system comes a wide range of services, including voice call, video call, and SMS texting, which can be transmitted via IP-enabled networks emerged. These digital networks are cheaper to build and operate than traditional circuit-switched networks. They have lower entry costs and are better suited to provide a range of improved, innovative, and economic service offerings to users of all kinds especially business and institutions (ITU, 2007). When VoIP is installed and used over a LAN (Local Area Network), it is free; you do not have to pay in order to use the services it provides but when used across internet in other to reach people more far from where you are you will need a service provider like MTN or Globacom to access the internet. VoIP is much cheaper than the PSTN and GSM.

This study seeks to add a VoIP technology over a traditional school local area network. This will consequently provide voice/video calls across the network. This will create a scalable LAN network for small business and organization and provide them with cheaper means of communication.

\section{Literature}

Since its inception, VoIP has opened new doors for telephony, bringing with it a multitude of new possibilities. The cost of VoIP is very low as compared to traditional telephony services, which is one of the key reasons for its success. The idea of voice over data stream enables VoIP to be transmitted and received using any IP-enabled computer, such as laptops, PCs, and Wi-Fi-enabled phones, etc. Voice over Internet Protocol (VoIP) use is projected to grow gradually, resulting in rapid network improvements. Various researchers have studied VoIP using various service groups in various network types. Here are a few examples of these works:

In their paper A Protected Voice over Internet Protocol (VoIP) Setup, Ogundile, Lawal, and Osanaiye (2012) demonstrated how to deploy a VoIP solution across a Metropolitan Area Network using mini Sip Server. The VoIP installation took place in a telecommunications training and research center in Lagos, Nigeria. The study shows that VoIP provides favourable environment, as it does not require much infrastructure as compared to the legendary PSTN. They also demonstrated in the study a suitable way of securing a VoIP network using VPN (Virtual Private Network) because it ensures privacy and integrity on traffic that traverse the organization internetwork and the internet.

Aiman Ahmed Abu Samra (2017) in paper, Performance Analysis of VoIP in WiFi Campus Network, simulated the performance of VoIP service in WiFi campus network using OPNET Modeler. He gradually increased the number of calls or workstations (WSs) in the network to investigate important Quality of Service parameters like jitter, packet end-to-end delay, wireless LAN load and wireless LAN throughput. Based on the simulation results, the study concluded that the capacity of the campus wireless LAN determines the access points and invariably the quality of the VoIP.

Jaclyn Packer and William Reuschel (2018), in paper, VoIP Accessibility: A Usability Study of Voice over Internet Protocol (VoIP) Systems and a Survey of VoIP Users with Vision Loss carried out a usability study and an online survey of VoIP users who are visually impaired. The survey examined the importance of common VoIP features, and both methods assessed difficulty in using those features. The method they used in carrying out the research includes four paid participants who are blind and four who have low vision. Four different tasks using four different VoIP systems (two Windows-based, two iOS-based) were presented in random order. The online survey included participants with prior VoIP experience: 50 individuals who were blind and 22 who have low vision. In conclusion they stated that the results of the online survey point to the importance of ensuring that VoIP systems are accessible to those with vision loss, and the results of the usability study demonstrate that VoIP soft phones can successfully be used by people with visual impairments.

\section{Materials and Methods}

This study simulates a voice and video communication (instant messaging) over a LAN network in other to reduce cost of communication from network providers and create a means of easy communication between staff members in an organization. This study includes two basic components which are; the software component and the hardware component.

The hardware component comprises two laptops, a phone, an Ethernet cable and a wireless router. One of the laptops is used for server installation; the other laptop serves as Client A while the Phone serves as Client B. The ObjectOriented Analysis and Design (OOAD) methodology and notation symbol of the Unified Modelling Language (UML) was used in the analysis of the system. Currently, the small and medium scale industry in Nigeria play a big role in the development of Nigeria and communication between individuals or staffs within this industry was done through PSTN (Public Switched Telephone Network) or GSM (Global System for Mobile Communications). This system was effective in 
carrying out voice communication over a network but at the same time cost much to establish and maintain. PSTN requires dedicated telephone lines from the service provider. In VOIP, one internet connection is all you need to transmit all voice channels.

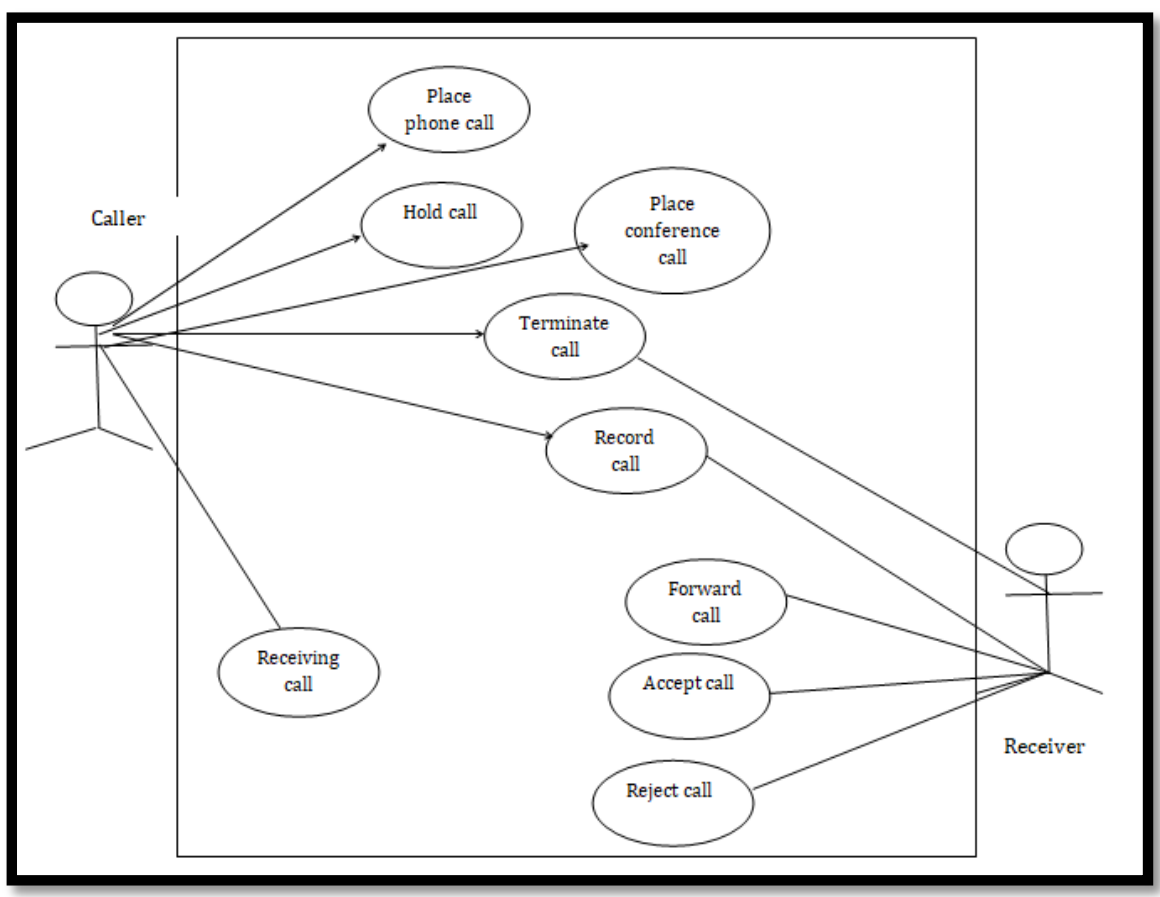

Figure 1: Use Case Diagram of the Proposed VOIP Network

\section{Results}

This section describes the implementation of the new system proposed in the previous section. The implementation involves the simulation of VoIPon a LAN as described in the analysis and design phase.

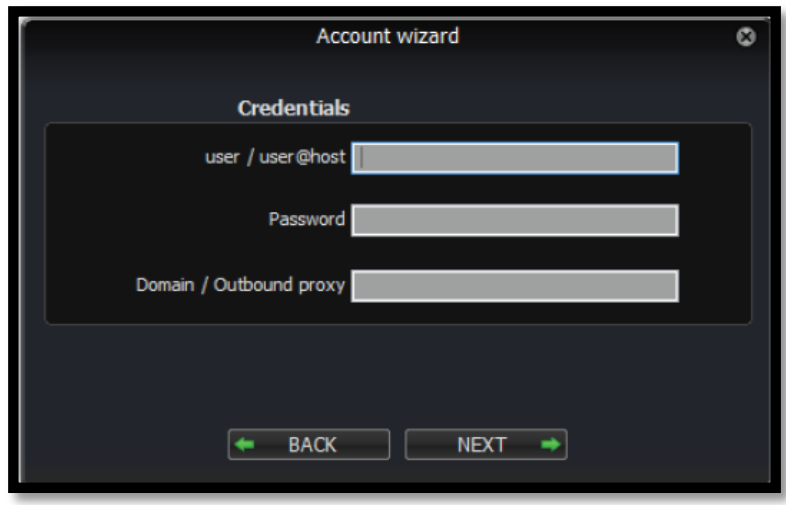

Figure 2: User Credential Interface.

The user is usually, prompted to select the account type he/she wants to configure. Then the system administrator provides a user with a username, password and possibly a hostname.

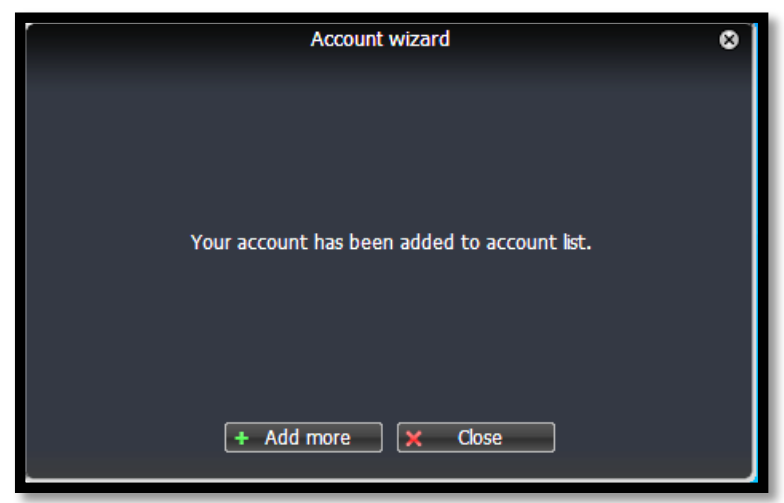

Figure 3: Access Grant Interface 
As soon as the user credentials have been confirmed, the system throws a confirmation message to the user as shown in figure 3 .

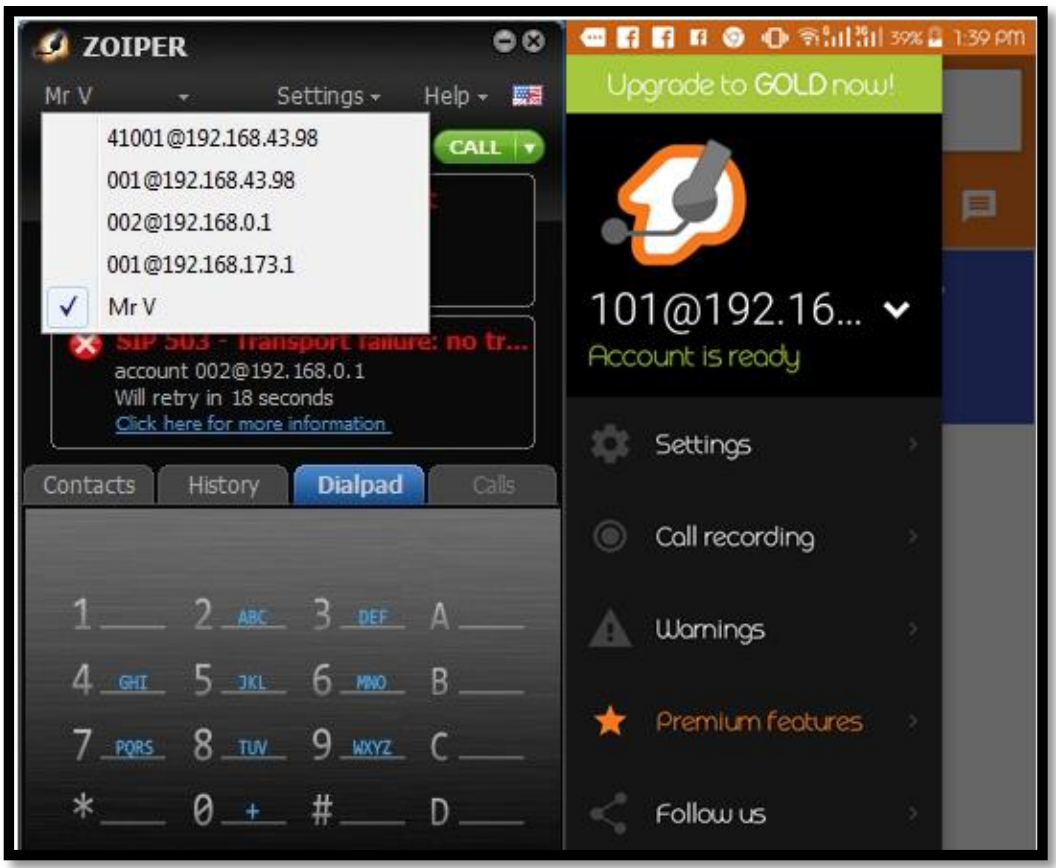

Figure 4: Interface for Client Software Connection Establishment for Both Phone and Desktop Application

Once the Zoiper network establishes a Connection with the mini SIP server it will indicate the particular IP address that is connected to the miniSIP server with a symbol $(\sqrt{ })$.

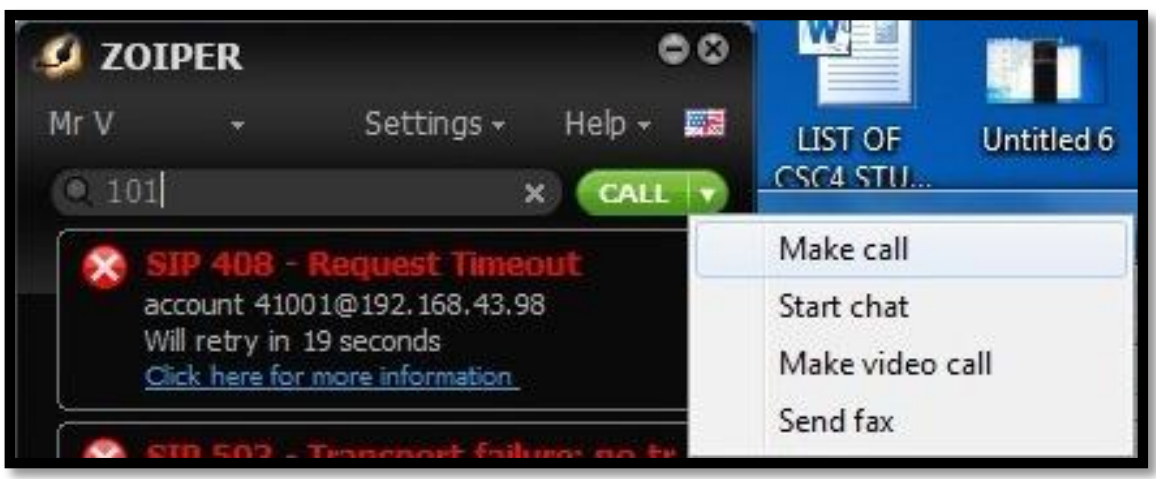

Figure 5: Zoiper Showing Call Type Selection Interface

Users can select the type of call they want to make whether voice or video call after dialing the number they want to call. In this case we dialed 101.

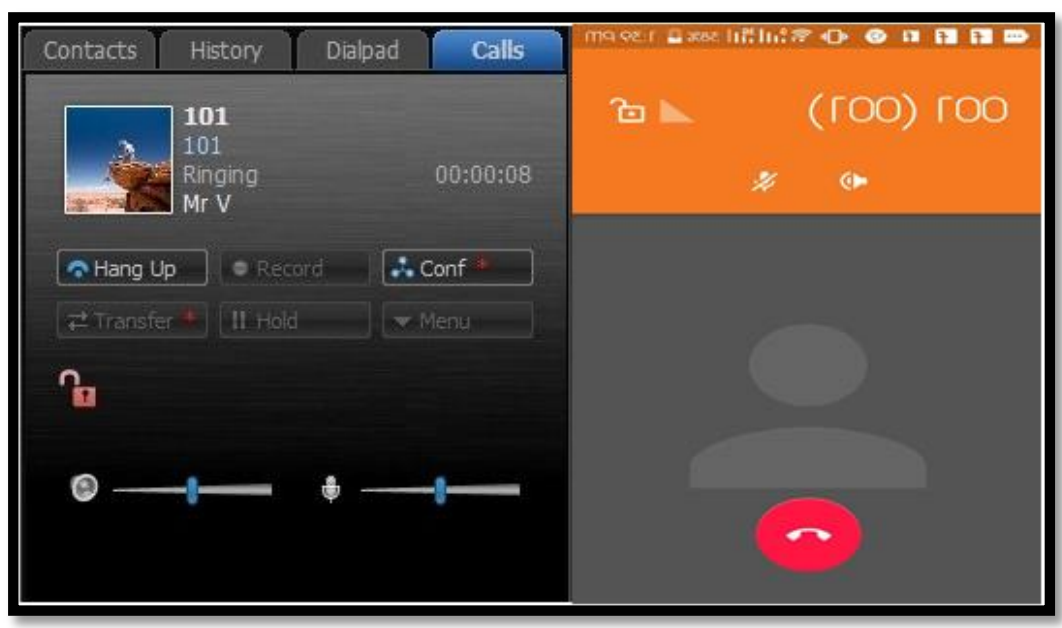

Figure 6: Zoiper Showing Call Interface for both Phone and Desktop 

from a phone.

In the figure above, client 101 is calling client 100. 101 is calling client 100 from desktop while client 100 received

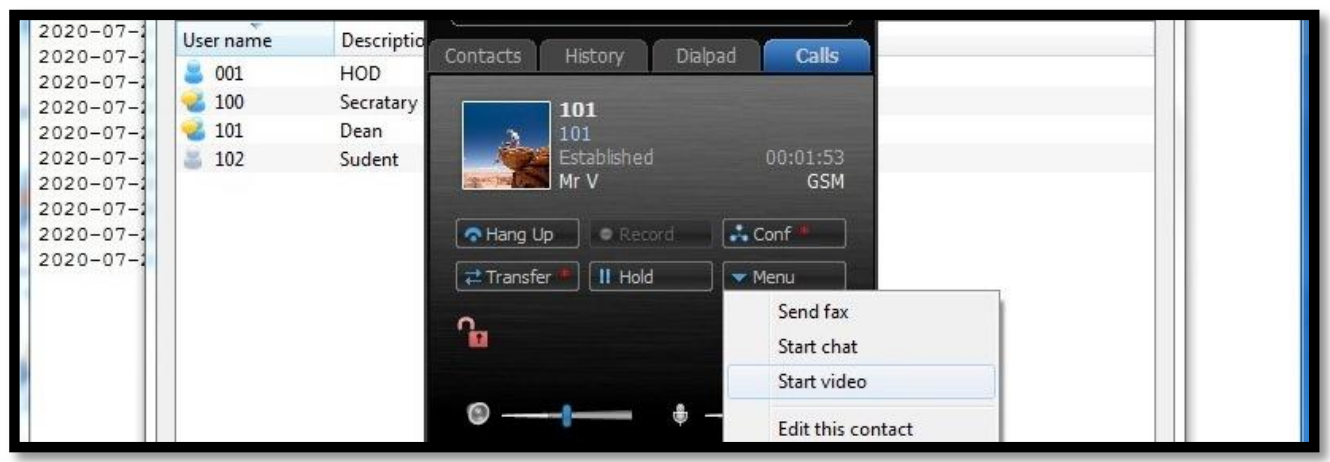

Figure 7: Interface for Video Call Initiation Option

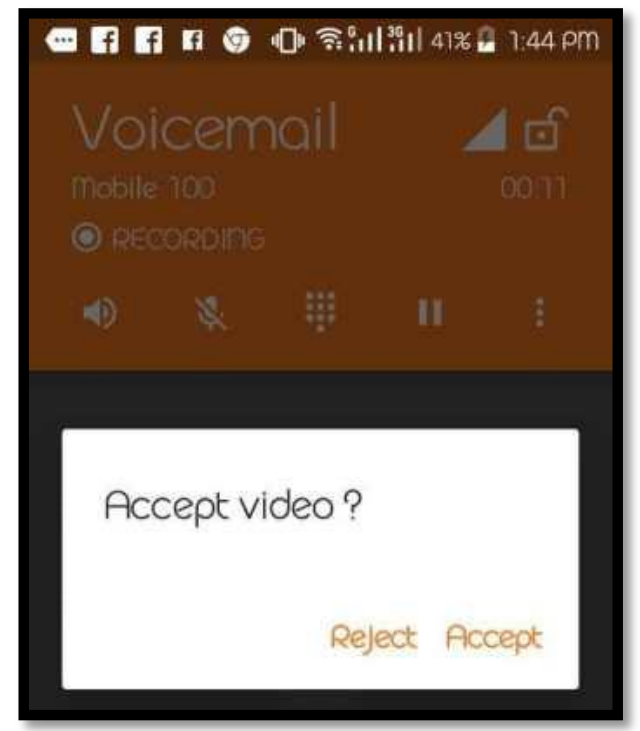

Figure 8: Interface for Video Call Acceptance or Rejection

When client a request for video call during voice call client B will be notified and he or she has the option to accept or reject the video call.

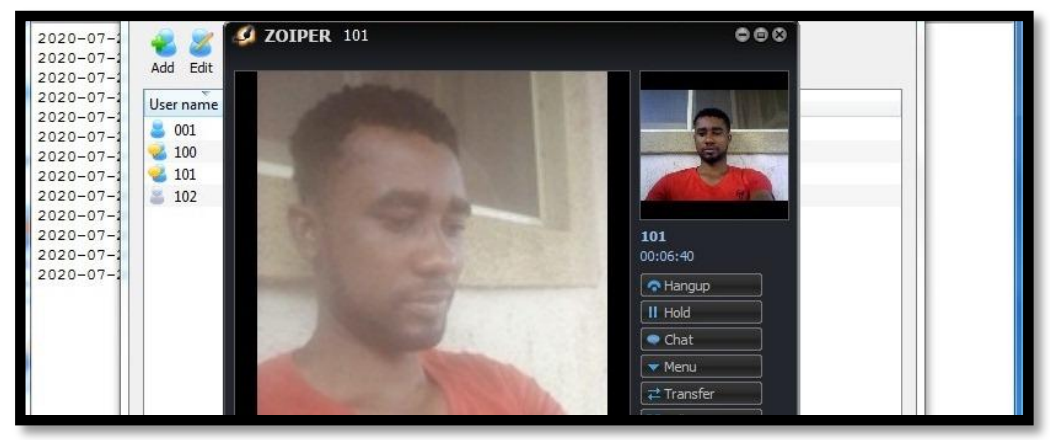

Figure 9: Video Call Interface for Both Desktop and Phone

Once client B accepts the video call request, it will automatically change from voice call to video call.

\section{Conclusion}

This study simulated VoIP over a LAN with the goal of implementing a Local area network telephony system using VoIP technology. A prototype design was specified and a real-time testing was done using some hardware devices, a miniSIP server and a Zoiper softphone to demonstrate that VoIP can be successfully deployed to provide in a flexible manner, additional data-driven services in Local area network telephony. In other for the system to perform effectively without failure from signal congestion, network links should be aggregated (bundled) for more bandwidth.

While this work had demonstrated the feasibility of leveraging on the low cost of IP-enabled telephone or softphone and developing a VOIP-based LAN network telephony, further research is recommended to test for voice quality and network performance as well as ability for network capacity planning when the system is fully implemented. 


\section{References}

i. Ahmed, A. and Abu S. (2017). Performance Analysis of VoIP in WiFi Campus Network. International Journal of Computer Applications, 174(3), 9-13.

ii. Packer, Jaclyn and Reuschel, William (2018) VoIP Accessibility: A Usability Study of Voice over Internet Protocol (VoIP) Systems and a Survey of VoIP Users with Vision Loss. Journal of Visual Impairment and Blindness. Vol 112. Pg 47-60

iii. Dave Roos (2008). "How Telephone Country Codes Work". Available online www.HowStuffWorks.com.

iv. Fasiku, A. I. (2018). Available online www.jsaer.com Review Article A Review of Voice over Internet Protocol. 5(7), 96-106.

v. Mohammed, H. A., Ali, A. H., \& Mohammed, H. J. (2013). The Effects of Different Queuing Algorithms within the Router on QoS VoIP application Using OPNET. International Journal of Computer Networks \& Communications, 5(1), 117-124.

vi. Mathivanan, A. A. (2012). MiniSIP as a Plug-in Available online http://www.divaportal.se/smash/get/diva2:565706/FULLTEXT01.pdf

vii. Zhang, H. Y., Homer, J., Einicke, G., \& Kubik, K. (2006). Performance comparison and analysis of voice communication over Ad Hoc network. Proceedings of the 1st IEEE International Conference on Wireless Broadband and Ultra Wideband Communications, AusWireless 2006, January, 74-110.

viii. Ogundile O., Lawal B. and Osanaiye O. (2012) A Secured Voice over International Journal of Scientific and Engineering Research 3(3) 